\title{
SEQUENCE PACKAGE ANALYSIS: A New Natural Language Understanding Method for Intelligent Mining of Recordings of Doctor-Patient Interviews and Health-Related Blogs
}

\author{
Amy Neustein, Ph.D. \\ Linguistic Technology Systems \\ 1055 River Road, Suite 1013 \\ Edgewater, New Jersey 07020 \\ U.S.A. \\ lingtec@banet.net
}

\begin{abstract}
Medical histories provide a rich resource for diagnoses and treatment. Similarly, consumers' blog postings on health-related topics offer unique data for medical researchers, practitioners, and pharmacologists. Nevertheless, speech and text analytic programs for mining recordings of patients' histories or consumers' blogs are compromised by the ambiguities, repetitions and ellipses in natural speech, which can be more pronounced when the speaker or blogger is discussing a medical problem. Conventional systems or programs limited to a set of key words and phrases cannot process speech as it actually occurs; if a speaker or blogger fails to use the word(s) found in the speech application's vocabulary, a poor statistical word match (or no match) is given. This paper shows how Sequence Package Analysis is informed by algorithms that can work with, rather than be hindered by, less than perfect natural speech for intelligent mining of doctor-patient recordings and blogs.
\end{abstract}

Keywords: blogosphere, data mining, medical interview, natural language understanding, sequence package analysis

\section{Introduction}

Sequence Package Analysis offers a new and advanced Natural Language Understanding (NLU) method whose parsing structures consisting of lexical units and prosodic features are designed to reflect in

addition to syntax some of the more complex semantic aspects of communication. A discussion of the methodological basis of Sequence Package Analysis (SPA), formulated by this author, first appeared in the International Journal of Speech Technology [1]. SPA has been referred to by AI researchers as among the more advanced parsing methods for "captioning the 
text to which data mining is applied" so as to better detect the subtleties of human emotions [2]. By relying on the entire sequence package - a series of related speaking turns and parts of turns - rather than on individual words or combination of words and their attendant stress patterns, SPA can enable a speech system to detect, among other things, the wide range of speakers' emotions in recordings of doctor-patient interviews and health-related blogs.

Because blogs constitute a rapidly expanding medium of communication, with tens of thousands of blogs created each day [3], blog experts, such as Technorati's founder and CEO, David Sifry, estimate the blogosphere to double in size once every six months [4]. While there is still lack of consensus among experts over the interconnectivity of blogs (some have found "a majority of blogs link sparsely or not at all with other blogs" or connectivity itself to be "overrepresented" by the "privileged subset of popular blogs," known as the 'A-list') [5], there is no dispute over the fact that blogs closely resemble the informality of conversational dialog, as opposed to the structured format of written text, and can thereby benefit from advanced NLU methods.

Experts in the field of text analytics have acknowledged that by working with unstructured data, blogs may pose a serious challenge for those who attempt to perform sentiment analysis of blog postings and their commentaries [6]. Pharmaceutical companies, for example, eager to gather market intelligence data about customer satisfaction with new drugs will turn to text analytic programs that measure the emotional content of blogs, known as their "tonal quality." Such programs, however, may be compromised by the same vagaries of natural language that can compromise speech systems.

At present, speech analytic programs that mine natural language dialog for signs of distress, frustration, anger, and a host of other human emotions, are still of very limited effectiveness. Since they simply match the speaker's natural language input against the program's list of keywords (that is, relying solely on word-spotting to process a speaker's input), they cannot process speech as it actually occurs; if a speaker fails to use the word(s) found in the speech application's vocabulary, the system is "stumped," and a poor statistical word match (or no match) is given for the natural language entry. These same limitations apply to programs that look for changes in prosody, such as a sudden elevation of inflection and/or pitch, insofar as prosodic patterns, like lexical entries, vary across populations of speakers. All in all, when specific keywords or changes in prosody are not found, the speech system can readily overlook important affective data that are critical to making accurate assessments about the attitude and affect of the speaker.

SPA offers a new methodological approach to surmount these inherent difficulties in both speech and text analytic systems. Relying on the sequence package in its entirety as the primary unit of analysis, SPA detects meaning and tonal quality in the timing, frequency and arrangement of certain components of dialog or blogs. SPA, which parses a conversation or a blog for its relevant sequence packages, extracts data that are then included in the speech engine's (or text analytic) output stream, yielding a better understanding of the emotional state of the speaker or blogger. Given that dialog and blogs more likely consist of a blend of sequences folding into one another, rather than a string of isolated keywords or phrases, one can plausibly argue that speech applications and text analytic mining programs equipped with SPA can better accommodate how people really talk. In particular, SPA-driven programs can better interpret convoluted, repetitive, and elliptical speech, often occurring when a speaker or blogger is seeking professional guidance for a highly distressing medical problem, than conventional speech systems or text analytic programs that are limited to a well defined set of key words and phrases.

\section{Methodology}

The way SPA adjusts to speech that is less than "perfect" is to offer a set of algorithms that can work with, rather than be hindered by, the ambiguities, ellipses, idioms, metaphors, colloquialisms, and the many other facets of natural language dialog. Ironically, SPA mines conversations and blogs to find the very sort of dialog and blog data that would have been discarded (or simply ignored) by most speech and text analytic systems as meaningless diatribes, unwieldy talk, or talk that is far too amorphous to grasp. And while some of these discarded data (such as the heightened occurrence of inter-sentential clausal connectives, multiple use of anaphors, idioms and metaphors, or deviations from normal in inter- and intra-utterance spacing) might appear relatively unimportant to speech and text analytic programs, these data can be very significant in properly interpreting the emotional content found in natural language dialog and blogs.

Using SPA, the author has designed a BNF (Backus-Naur Form) table consisting of 60 (sixty) Sequence Packages - a typology of parsing structures representing the semantic aspects of communication - 
that capture the affective data found in natural speech and blogs. It is no easy task to formally map out the conversational sequence patterns of natural language dialog and blogs that reflect labyrinthine, and sometimes confounding, human emotions. To do this, SPA draws from the field of conversation analysis as its methodological basis. What conversation analysis provides is a rigorous, empirically-based method of recording and transcribing verbal interactions by using highly refined transcription signals to identify both verbal components and paralinguistic features, such as stress, pauses, gaps, overlaps and changes in intrautterance spacing [7].

By breaking down natural language communication into its elemental form of conversational sequences and speaking turns within those sequences, rather than isolated sentences or utterances, conversation analysts have been able in their 35 years of study of interactive dialog to identify and describe how participants in a dialog systematically accomplish their interactive work, while they are continually engaged in the process of making sense of the ongoing social activity. This is done by examining how speakers demonstrate, through the design of their speaking turns, their understanding and interpretation of each other's social actions, including the wide range of emotions embedded within those actions, such as a speaker's noticeable failure to answer a question directed at him or her, which in certain instances may indicate annoyance or irritation with the other speaker, rather than failure to hear the question.

Reduced to algorithms, many sequence packages are naturally transferable from one contextual domain to another, which means that many of the same sequence package structures found in the conversations of doctors and patients or in patient's blogs, also appear in call center dialog between distressed customers and call center agents. In addition, by focusing on social action, rather than on grammatical discourse structure solely, this new NLU method for mining conversations and blogs can potentially be applied to a myriad of other languages, including Arabic and Farsi, because "all forms of interactive dialog, regardless of their underlying grammatical discourse structures, are ultimately defined by their social architecture" [8].

\section{Design}

There are two ways that an SPA-driven speech or text analytic mining program can work. First, it can serve as an "add on" layer for conventional data mining programs, including those built on vector- based models, which assign n-grams and bi-grams and hold spaces in between words and word phrases accordingly. If SPA functions as an "add on" layer, the "global weighting" to be applied for the next layer of analysis need no longer be limited to content words or their term roots; rather, it can now also encompass sequence package material. To accomplish this, SPA uses Statistical Language Modeling (SLM) - the standardized method for matching speech input to the speech application vocabularies - but instead of generating candidate words and word phrases for the speech input, SPA generates candidate sequence packages. Thus, using the same method of weighting possibilities used for candidate words and word phrases, SPA detects the range of possible sequence packages present at each stage of the conversational sequence, the totality of which makes up the dialog [9].

As an "add on" layer, SPA can take the output of a speech engine and provide a deeper level of analysis of the patient's dialog or blog message by interpolating sequence package information into the output stream. By marking sequence package boundaries and specifying package properties, the SPA-enhanced mining program gives the software downstream the contextual indicia - the precise location points in the flow of interactive dialog, which signify the different conversational activities and phases of the dialog - needed to interpret the rest of the data stream reliably.

Given that much of blog material, likewise, consists of different phases, including but not limited to narrative complaints, digressions, and complaint resolution, it is essential that a text analytic program, in addition to taking note of the descriptors used by the blogger, provide contextual indicia for analyzing the tonal quality of blogs. For example, since the more strident tonal features are most likely to occur in the digressive phase of the blog, as opposed to the narrative complaint or the complaint resolution phase, a program designed to identify the different phases of blogs will likely give less credence to product criticism occurring in the digressive phase, where diatribes and rants are to be expected. Thus, failure to isolate the various phases of the blog can quite plausibly skew sentiment analysis, by ascribing undeserved importance to the descriptors found in the digressive portion of the blog [10].

Second, SPA might be used as a wholly integrated system rather than as an "add on" layer to conventional speech and text analytic programs. In such a case, such programs would use sequence package grammars, represented by the specially designed BNF table of parsing structures, rather than words and word phrases as their starting point. Such a 
use would allow the building of an entire vocabulary, by methodically uncovering the keywords characteristically embedded within these sequence package templates, without necessarily having to have an a priori knowledge of the words and word phrases in the speech application.

But regardless of whether SPA is built into a system as an "add on" layer of intelligence or in the alternative as a wholly integrated system, it can be argued that SPA, for the most part, can enhance the scalability of data mining and text programs. This is so because SPA can help to streamline the corpus of data required to build a statistical language model, by focusing on commonly occurring sequence packages that are generic to a large population of speakers and bloggers, and thereby eliminate the need to construct elaborate speech application vocabularies, in anticipation of every possible word to be used by a speaker or blogger.

\section{Doctor-Patient Interviews}

Medical history-taking provides the physician with one of the richest resources for making accurate diagnoses and instituting proper treatment. And in our cost-conscious world, medical histories take on an even greater value: by getting a complete picture right at the beginning, doctors can cut back on the many unnecessary and costly tests that are given to patients to provide the clinician with the necessary information that the patient failed to provide in his history. But ironically, what should be a relatively simple task is often complicated by so many conversational difficulties that the value of the history is minimal. Several things contribute to these conversational problems.

First, doctors have large loads and very little time to spend with each patient. Working under such constraints, it becomes hard to listen to each patient, especially to those who are long winded. Second, patients all too often present their medical problems and histories in such a roundabout way that they leave their doctors with more questions than answers. Third, patients increasingly change providers, as their health plans are determined by their jobs, relocation, marriage/divorce, and other demographic factors. As a result, their care providers don't have the opportunity to really get to know them as the old fashioned doctors once knew their patients.

One solution to these difficulties is intelligent mining of tape recordings of doctor-patient interviews. These are used to uncover important medical history data that escapes the practitioner's attention, especially when patients are circuitous and vague. Intelligent mining can also uncover the emotive content of the medical interview in which patients might express their fears and concerns in such indirect ways that it escapes the notice of the physician altogether. Such mining can be done in real time during the medical visit or afterwards, so that feedback can be given to the doctor for a follow-up visit. Either way, intelligent data mining can extract important diagnostic data that is submerged beneath layers of dialog. In addition, it can uncover the patient's fears and worries. The payback would then be twofold: 1) more efficient handling of the case; and 2) an improvement in patient satisfaction.

Here is an illustration of doctor-patient dialog, derived from tape-recorded interviews of patients at a New York teaching hospital. In this example, which is reflective of a common pattern, the patient offers very important history data to the physician at the wrong place in the medical encounter, when the doctor's attention has been diverted to something else. The patient, despite ample opportunity to give this vital history data when the doctor was looking for it, delayed mention of these important facts - presumably fearing that such facts would portend a serious diagnosis for her. In this example, vital family medical history about bone cancer was withheld when the physician queried the patient about family history, causing the doctor (questioned by this author) to either minimize or ignore the importance of this history datum when given later on in the interview. It is beyond question that such delayed patient disclosures - no matter how critical to formulating an accurate diagnosis and an effective treatment plan - can go over the doctor's head. Inasmuch as it is the doctor and not the patient who directs the questioning in the medical interview [11], it is the doctor who likewise directs the "listening" in the medical interview. As a result, doctors can selectively "tune out" at times, particularly when patients give information at the wrong place in the interview.

Patient: "I become terribly worried about my pain, which reminds me of the arthritic pain that my sister had, which turned out to be bone cancer, so I worry whenever I have pain because I don't know if it is what she had."

In this case, the patient made this revelation about a family history of bone cancer when the physician was busy conducting his physical examination. Ironically, a few minutes earlier, when the doctor during the medical interview had asked specifically about any history of family illnesses, the patient was 
silent. However, one must look closely at how the patient actually relayed this information about her family history. What is striking is that when the patient revealed the family history, she obfuscated her overriding medical concern - which was whether her own pain could reflect an undiagnosed case of osteosarcoma (bone cancer) - by embedding it in a series of narrative type statements, as if she were telling a story to a neighbor or friend. By revealing this medical history information in the form of a narrative, and at a juncture in the examination where medical history questions were no longer topical, the patient possibly hoped her fear of bone cancer would be given less attention than if she came out with it directly when asked about her family history. Yet, at the same time, the fact that the patient brought up her sister's bone cancer at all indicates that she wanted the doctor to address her concerns and allay her fears.

Although this patient straddled the fence, using dialog that conveyed the importance of this history datum while at the same time trying to downgrade its impact, an SPA-driven mining program could retrieve from the recording of this interview this otherwise "lost" data, and its strong emotional implications for the patient.

Here's how. First, SPA would detect a high usage of narrative phrases in close proximity to one another by matching the parsing structures consistent with "troubles-related narration" against the speech input derived from the recording of the doctor patient interview. The arrangement of parsing structures forms the following sequence package, which is divided into four parts. The narrative predicates appear here in bold italics:

1) a short, condensed and somewhat nonspecific concern preceded by a narrative phrase

\section{I become terribly worried about my pain}

2) an expansion of the concern, citing the troublesome datum ("bone cancer"), which is embedded within two narrative predicates

which reminds me of the arthritic pain that my sister had

which turned out to be bone cancer

3) a recycling of the nonspecific concern preceded by a narrative phrase

so I worry whenever I have any pain
4) a reference back to the expanded concern, but only with the use of pronouns that serve as anaphors, referring back to the expanded concern

because I don't know if it is what she had

Second, upon finding sequences that are punctuated by a high amount of narrative predicates, in combination with an elevated use of anaphors, the SPA-driven mining program would activate a heuristic procedure for discerning from this vague and winding dialog the true nature of the patient's complaint. To do that, SPA grammars would search for the specific content material (arthritic pain and bone cancer) embedded within the two narrative predicates ("which reminds me of...which turned out to be...") appearing in the second part of this four-part sequence package. In addition, such grammars would uncover those specific content words ("worried") and their term roots ("worry") - associated with a high emotive index - upon locating this narrative sequence package in the patient's dialog. At the end, the SPA mining program would offer the physician the information that this patient has bone cancer in her family and that she is quite concerned that she too might be stricken with bone cancer, just as her sister was. Such information might help the physician to make the decision to order periodic bone scans and MRIs so as to keep a careful watch for the possible development of osteosarcoma, a disease that may be more successfully treated if caught in its early stage(s) of development.

\section{Health-Related Blogs}

Consumers' blog postings and comments on health-related topics present a goldmine of data for medical researchers, practitioners, and pharmacologists. Nowhere can one find such immediate feedback on new drugs and medical devices than in the blogosphere. Ironically, those who eschew surveys, refusing to answer questionnaires handed to them at their doctor's office or received in the mail, will freely post their personal data on blog sites without giving it a second thought.

When applying SPA to the blogosphere, the same methodological approach used to analyze natural speech is applied to blogs. Drawing from the BNF table of parsing structures that represent the semantic aspects of communication, and their attendant affective features, an SPA-driven text analytic program begins by marking off sequence packages 
that indicate the different phases of the blog, such as narrative complaint, digression, and complaint resolution. To assess the true tonal quality of the blog, a score, based on the frequency and intensity of adjectival descriptors, is generated for each phase respectively, before calculating the final tonal quality score. Here is an example:

A blogger, who had been taking Ambien, a frequently prescribed sleep inducing agent, posted her reactions to this drug on a popular blog site. The blogger used strong, inflammatory language, filled with adjectival descriptors that connoted anger and frustration. However, on closer look it turns out the blogger reserved her strong emotive descriptors for the "digressive" portion of the blog, rather than her narrative complaint or complaint resolution. Since conventional text analytic programs commonly add up the total number of descriptors in the blog, without first marking off the sequence packages that differentiate, for example, between digressions, narrative complaints, and complaint resolution, sentiment analysis can be distorted by the excessive use of strong descriptors in the digression phase. In this example, in contrast, SPA would minimize (or even discount) the potency of the language used in the digression phase, focusing more accurately on the tonal quality of the narrative complaint and the complaint resolution. As a result, the total score given for the blog's tonal quality would be much closer to the true affective state of the blogger. In so doing, SPA enables text analytic programs to perform more accurate sentiment analysis, which in turn produces more reliable market intelligence data.

\section{References}

[1] Amy Neustein, "Using Sequence Package Analysis to Improve Natural Language Understanding," International Journal of Speech Technology, 4(1), 2001, pp. 31-44.

[2] M. Paprzyki, A. Abraham, and R. Guo, "Data Mining Approach for Analyzing Call Center Performance," The $17^{\text {th }}$ International Conference on Industrial Engineering Applications of Artificial Intelligence and Expert Systems. Ottawa, Canada. In Lecture Notes in Computer Science. Germany: Springer Verlag, 2004.

[3] A. Aschenbrenner and S. Miksch, "Blog Mining in a Corporate Environment," Vienna University of Technology, Institute of Software Technology and Interactive Systems and Research Studios Austria, Smart Agent Technologies, Vienna Technical Report, Asgaard-TR, November 2005.

[4] David Sifry, "State of the Blogosphere," Sifry's Alerts, August 7, 2006.
[5] S. Herring, et al., "Conversations in the Blogosphere: An Analysis "From the Bottom Up,", Proceedings of the $38^{\text {th }}$ Annual Hawaii International Conference on System Sciences. Los Alamitos: IEEE Computer Society Press, January 3-6 2005.

[6] "Exploring Attitude and Affect in Text: Theories and Applications," AAAI Spring Symposium. Stanford University, March 22-24, 2004.

[7] J.M. Atkinson and J. Heritage, Transcript notation. In J.M. Atkinson and J. Heritage (Eds.), Structures of Social Action: Studies in Conversation Analysis. Cambridge University Press, Cambridge, 1984, pp. ix-xvi.

[8] Amy Neustein, "Sequence Package Analysis: A New Global Standard for Processing Natural Language Input?" Globalization Insider, vol. X111(1, 2), February 18, 2004.

[9] Amy Neustein, "Using Sequence Package Analysis as a New Natural Language Understanding Method for Mining Government Recordings of Terror Suspects," Proceedings of the 3rd International Workshop on Natural Language Understanding and Cognitive Science, ICEIS 2006, Paphos, Cyprus, pp. 101-108, May 23-24, 2006.

[10] Amy Neustein, "Performing Sentiment Analysis of Blogs,” Linguistic Technology Systems, April 2006.

[11] Rich Frankel, "Talking in Interviews: A Dispreference for Patient-Initiated Questions in Physician-Patient Encounters." In G. Psathas (Ed.), Interaction Competence. Washington, D.C.: University Press of America, 1990, pp. 231-262. 